\title{
Synchronous and Antecedent Non-Thyroidal Malignancies in Patients with Papillary Thyroid Carcinoma
}

\author{
Sara Murray, MD, David F Schneider, MD, MS, Philip S Bauer, MS, Rebecca S Sippel, MD, \\ FACS, and Herbert Chen, MD, FACS \\ Section of Endocrine Surgery, Department of Surgery, University of Wisconsin, Madison, WI
}

\begin{abstract}
Background-There is a known association between the development of papillary thyroid cancer (PTC) after a primary non-thyroidal cancer (NTC). However, the prevalence of synchronous or antecedent NTCs in patients with PTC is undetermined, as are the clinicopathologic characteristics of PTC in these patients.
\end{abstract}

\begin{abstract}
Study Design-A review was performed of our prospectively maintained PTC database between January 1995 and December 2010. Information collected included patient and tumor characteristics, past medical history, PTC presentation, and treatment modality.
\end{abstract}

\begin{abstract}
Results-433 adult patients underwent thyroid resection for PTC. 67 cases of synchronous or antecedent NTCs were observed in 60 patients (13.9\%). The most commonly associated antecedent NTCs were breast $(n=11)$, prostate $(n=8)$, and melanoma $(n=5)$, while renal cell carcinoma $(n=3)$ and melanoma $(n=3)$ were the synchronous NTCs most observed. Compared to patients without an NTC, those with an NTC were older (56.4 \pm 15.5 vs $44.9 \pm 14.2$ years, $\mathrm{p}<0.0001)$, experienced prior radiation exposure ( $35.0 \%$ vs $3.5 \%, \mathrm{p}<0.001)$, and more commonly presented with a thyroid mass incidentally on imaging $(41.7 \%$ vs $9.1 \%, \mathrm{p}=<0.001)$. PTC tumor characteristics were similar between groups, except that NTC patients presented at a more advanced stage. However, when analyzed independently, primary tumor size, and nodal and distant metastases were comparable.
\end{abstract}

Conclusions-The prevalence of synchronous or antecedent NTCs in patients surgically treated for PTC is $13.9 \%$. These patients present with similar PTC tumor characteristics as those without additional NTCs, and should therefore be managed equivalently. In addition, surgeons should be aware of the frequency of synchronous PTC with these types of tumors and consider evaluation of the neck at the time of NTC diagnosis.

\section{Introduction}

Thyroid cancer is the most common endocrine malignancy, and the annual incidence has risen nearly $50 \%$ since the 1970 s $(1,2)$. The $2005-2009$ age-adjusted annual incidence rates

\footnotetext{
(C) 2013 American College of Surgeons. Published by Elsevier Inc. All rights reserved.

Correspondence address: Herbert Chen, M.D., FACS, University of Wisconsin, Department of Surgery, Section of Endocrine Surgery, K3/705 Clinical Science Center, 600 Highland Avenue, Madison, WI 53792, USA, Tel.: 608-263-1387, Fax: 608-263-7652, chen@surgery.wisc.edu.

Disclosure Information: Nothing to disclose.

Abstract presented at the American College of Surgeons 98th Annual Clinical Congress, Surgical Forum, Chicago, IL, October 2012.

Publisher's Disclaimer: This is a PDF file of an unedited manuscript that has been accepted for publication. As a service to our customers we are providing this early version of the manuscript. The manuscript will undergo copyediting, typesetting, and review of the resulting proof before it is published in its final citable form. Please note that during the production process errors may be discovered which could affect the content, and all legal disclaimers that apply to the journal pertain.
} 
are 17.3 per 100,000 women and 5.9 per 100,000 men according to the Surveillance Epidemiology and End Results (SEER) cancer registry (1). Interestingly, the incidence of follicular, anaplastic, and medullary thyroid cancer have not significantly changed over time, and virtually all of the observed increase is attributable to a rise in papillary thyroid cancer (PTC) (2).

PTC is the most common form of thyroid cancer, comprising nearly $90 \%$ of all thyroid cancers (2). Known risk factors for PTC include exposure to ionizing radiation at a young age, and a history of thyroid disease, primarily benign thyroid nodules, and potentially Hashimoto's thyroiditis (3-5). Familial cancer syndromes such as Cowden's syndrome and familial adenomatous polyposis are also associated with PTC $(6,7)$. Numerous etiologies have been proposed for the rise in PTC incidence and include hormonal factors, changes in histologic criteria, and increased diagnostic scrutiny $(2,8-11)$. In addition, several studies have reported an increased incidence of thyroid cancer after a primary malignancy at multiple sites (12-15). This association might be related to treatment effects of the primary malignancy, specifically radiation therapy; however, some studies have noted an increased risk of thyroid cancer as a second primary after other cancers in patients without prior radiation therapy $(12,16,17)$. Furthermore, many studies have reported an increased rate of secondary malignancies among thyroid cancer survivors, suggesting a 2-way positive association (12-14,18-20).

The majority of existing literature on this topic largely focuses on the risk of developing various cancers after a primary thyroid cancer, and to a lesser extent, the risk of thyroid cancer after a prior non-thyroidal malignancy. However, to our knowledge, no studies have examined the clinicopathologic characteristics of PTC in patients with an additional malignancy. Therefore, to further our understanding of this association, the objectives of this study were the following: 1) determine the prevalence of an additional synchronous or antecedent non-thyroidal cancer (NTC) in patients surgically treated for PTC, and 2) compare the clinicopathologic characteristics of PTC between patients with and without an additional synchronous or antecedent NTC.

\section{Methods}

All patients who undergo thyroid surgery at our institution are documented in a prospectively maintained database. The current study is an Institutional Review Board approved retrospective review of patients that underwent a thyroid resection at our institution between January 1995 and December 2010, and had PTC on final pathology. Exclusion criteria included patients less than 18 years of age and patients with a history of thyroid resection for PTC prior to their presentation at our institution, unless the detailed pathologic information for their initial surgery was available in our electronic medical record. If this were the case, the pathology from the initial surgery was used for analysis. Patients with follicular variant PTC were included. In addition, patients with a history of antecedent non-PTC thyroid cancer $(n=7)$ were included but considered part of the "without NTC" group during analysis.

Information contained in our database and utilized for this study included the following: patient demographics, medical history including synchronous or antecedent malignancies, surgical history, previous radiation exposure, clinical presentation of PTC, indication for thyroidectomy, pathologic information, treatment intervention(s) for PTC, follow-up, and survival. Regarding pathologic information, papillary thyroid microcarcinoma was defined as the largest focus of PTC in the pathologic specimen measuring $1 \mathrm{~cm}$ or less in diameter. Furthermore, the tumor-node-metastasis (TNM) staging system of the American Joint Committee on Cancer (AJCC) was used for tumor classification of PTC (21). Similar to 
prior studies, it was assumed that patients without evidence of lymph node metastases on pre-operative ultrasound, pathologic analysis, or ${ }^{131}$ I post-therapy scintigraphy were negative for lymph node metastases (N0) $(22,23)$.

Synchronous malignancy was defined as the diagnosis of an NTC within 6 months of PTC diagnosis. Antecedent malignancy was defined as the diagnosis of an NTC greater than 6 months prior to PTC diagnosis. The latency period between the two diagnoses was calculated from the date of primary NTC diagnosis to the date of PTC diagnosis by fine needle aspiration (FNA). If the date of diagnostic FNA biopsy was unknown, or if the patient did not have a pre-operative diagnosis of PTC, the date of thyroid resection was used as the date of PTC diagnosis. If a patient had multiple antecedent NTCs, only the initial cancer was utilized for latency analysis. The follow-up period for all patients in the series was calculated from the time of thyroid surgery to the most recent clinic visit by either the operating surgeon or the endocrinologist.

Data were analyzed using Stata version 12 software (StataCorp, College Station, TX), and are expressed as either mean with standard deviation for continuous, normally distributed variables, or as the median with interquartile range (IQR) for non-normally distributed variables. Comparisons of continuous variables were made using Student's T-test, whereas comparisons of categorical variables were performed using chi-squared or Fisher's exact test when appropriate. Values of $\mathrm{p}<0.05$ were used to determine statistical significance.

\section{Results}

\section{Clinicopathologic characteristics of PTC in patients with synchronous or antecedent malignancy}

A total of 433 patients with PTC were included in this analysis. The mean age was $46.5 \pm 14.9$ years and $73.7 \%(n=319)$ were female. 60 patients $(13.9 \%)$ had either a synchronous or antecedent NTC. Table 1 outlines the patient and tumor characteristics of patients with and without a synchronous or antecedent malignancy. Compared to patients without an NTC, patients with an NTC were older $(56.4 \pm 15.5$ vs $44.9 \pm 14.2$ years, $\mathrm{p}<0.0001)$, experienced prior radiation exposure ( $35.0 \%$ vs $3.5 \%, \mathrm{p}<0.001)$, and more commonly presented with a thyroid mass incidentally on imaging $(41.7 \%$ vs $9.1 \%$, $\mathrm{p}=<0.001)$. Though patients with an NTC were diagnosed at a more advanced stage of disease than those without ( $\mathrm{p}=0.001$ ), when analyzed as individual TNM categories, pathologic features including primary tumor size and the presence of lymph node or distant metastases were similar between the groups (Table 1). The incidence of papillary thyroid microcarcinoma was equivalent, as were histologic features such as multifocality, extrathyroidal extension (ETE), and aggressive variants. Furthermore, the treatment methods for PTC were similar despite the presence of a synchronous or antecedent NTC (Table 1).

Of note, of the 190 patients with microcarcinomas, 62 (32.6\%) were incidentally discovered after thyroidectomy was performed for an alternate indication, including an enlarging or symptomatic nodule/goiter, indeterminate FNA, Graves' disease. Surgical treatment consisted of a total or completion thyroidectomy in $163(85.8 \%)$ of these patients, and there was no difference in the rate of total or completion thyroidectomy between patients with and without an additional NTC ( $85.3 \%$ vs $88.9 \%$, $\mathrm{p}=0.77)$.

\section{Types of NTC}

Table 2 outlines the specific types of NTC observed in this series. As shown, there were 67 observed cases of synchronous or antecedent NTCs occurring in 60 patients (13.9\%) with PTC. Seventeen patients (3.9\%) had a synchronous NTC, and 47 patients (10.9\%) had an antecedent NTC. Four patients had both a synchronous and antecedent NTC, 1 patient had 2 
synchronous NTCs, and 2 patients had 2 antecedent NTCs, accounting for the 67 cases. The 4 patients with both synchronous and antecedent cancer have each primary tumor listed independently in the corresponding timing of occurrence in Table 2, and the patients with more than one synchronous or antecedent cancer have each pathology displayed separately as well. Overall, the most commonly observed NTCs were breast $(n=12)$, prostate $(n=9)$, melanoma $(n=8)$, leukemia $(n=6)$, and lymphoma $(n=4)$. The most frequently observed synchronous NTC were renal cell carcinoma $(n=3)$, melanoma $(n=3)$, and oral cavity/ pharyngeal cancers $(n=2)$.

\section{Timing and Presentation of PTC}

Of the 17 patients with a synchronous NTC, 16 were diagnosed with PTC upon workup for their primary NTC. The mean latency period between NTC and PTC diagnosis was 2.7 months (median=2.4; IQR, 1.2-4.2). In these patients, PTC most commonly presented as an incidental mass on imaging $(\mathrm{n}=9,56.3 \%)$, followed by a palpable neck mass $(\mathrm{n}=5,31.2 \%)$. Two patients (12.5\%) had PTC discovered incidentally during neck surgery for their NTC. Alternatively, 1 patient in the synchronous NTC group was initially diagnosed with PTC after palpation of a neck mass, and was subsequently found to have a synchronous NTC. During the pre-operative evaluation for PTC, the patient complained of nausea and abdominal pain. After the appropriate work-up, the diagnosis of a gastrointestinal stromal tumor and concomitant pheochromocytoma was made 1.9 months after PTC diagnosis.

Of the 47 patients with an antecedent NTC, the mean latency period to PTC diagnosis was 11.3 years (median $=8.5 ; \mathrm{IQR}, 3.9-16.8)$. The majority $(\mathrm{n}=35,74.5 \%)$ were diagnosed with PTC independently of surveillance for their primary NTC. Patients typically presented with PTC after palpation of a neck mass $(\mathrm{n}=24,51.1 \%)$, followed by an incidental mass on imaging $(\mathrm{n}=18,38.3 \%)$, incidentally at the time of surgery for alternate pathology (e.g. Hashimoto's thyroiditis) $(n=4,8.5 \%)$, or cervical lymphadenopathy $(n=1,2.1 \%)$.

While patients with an antecedent or synchronous non-PTC thyroid malignancy $(n=7)$ were included in the "without NTC" group for this analysis, it should be noted that 4 had a pathologically independent non-PTC thyroid malignancy simultaneously with PTC (follicular thyroid carcinoma $[\mathrm{FTC}]=2$, insular carcinoma $=1$, anaplastic carcinoma $=1$ ), and 3 patients had an antecedent FTC and thyroid lobectomy prior to presenting with PTC.

\section{Follow-up}

The mean length of post-operative follow-up was 5.1 \pm 4.1 years (median=4.1; IQR, 2.0-7.3). Patients without an NTC had a longer mean follow-up than those with an NTC $(5.3 \pm 0.2$ vs $3.8 \pm 0.3$ years, $\mathrm{p}=0.007)$. Thirteen patients $(3.0 \%)$ died during the follow-up period, 7 $(53.8 \%)$ of which had an NTC. The types of NTC in the deceased patients included the following: prostate cancer $(n=3)$, melanoma $(n=1)$, lymphoma $(n=2)$, leukemia $(n=1)$, and esophageal cancer $(\mathrm{n}=1)$. One patient had a history of both prostate cancer and lymphoma. The mean survival in these 7 patients was $2.9 \pm 1.9$ years (median=2.6; IQR, 1.8-4.8), compared to the 6 patients without an NTC who survived a mean of $6.3 \pm 5.4$ years (median=4.5; IQR, 3.0-11.8) $(\mathrm{p}=0.15)$. Two patients eventually died of their NTC (esophageal and prostate cancer).

Overall, 5 patients died from thyroid cancer at a mean of 2.2 \pm 2.1 years (median=3.0; IQR, 0.13-3.3) after PTC diagnosis. Three of these 5 patients had a primary antecedent NTC (leukemia, lymphoma, and prostate cancer), and presented with stage IV, III, and I PTC, respectively. None had aggressive forms of PTC, however, the patient with stage I PTC was successfully cured of disease but 4.4 years later developed anaplastic thyroid cancer in the 
lymph nodes. The 2 remaining patients without an NTC died of recurrent PTC and concomitant PTC/anaplastic thyroid cancer, respectively.

\section{Discussion}

Our results show a $13.9 \%$ prevalence of synchronous or antecedent NTC in patients surgically treated for PTC, with the most commonly associated malignancies being breast, prostate, melanoma, leukemia, and lymphoma. This study was novel in that it examines the clinicopathologic features of PTC in these patients. Significant differences observed between the groups included patients with an additional NTC being older, with previous radiation exposure, and more likely to present with a thyroid mass incidentally on imaging. Regarding PTC pathology, patients with an NTC had similar primary tumor characteristics on presentation when compared to those without, with the exception of stage at presentation. Although patients with an NTC were diagnosed at a more advanced stage of disease than those without, independent analysis of each TNM category demonstrated no significant differences in the primary tumor size, or the rate of nodal or distant metastases between the groups. This discrepancy in stage is likely attributable to the AJCC TNM staging system which stages patients $\geq 45$ years of age at a more advanced stage of disease than patients $<45$ years. Given the significantly older average age of patients with an additional NTC, the stage at diagnosis is correspondingly higher, despite similarities in primary tumor size and the presence of nodal or distant metastases between the groups. Additional histologic features including the presence of multifocality, ETE, and aggressive variants were comparable between the groups.

The association between thyroid cancer and other malignancies has been well documented in the literature, with the majority of these studies examining the risk of a secondary malignancy after thyroid cancer (12-14,18-20). However, several studies have evaluated the reciprocal risk of developing a secondary thyroid cancer succeeding other malignancies (1215). In a cohort of over 2 million cancer survivors, Ronckers et al. (12) utilized the SEER cancer registry to demonstrate a $42 \%$ overall increased risk of developing thyroid cancer after a primary NTC compared to the general population, based on 1,366 cases of secondary thyroid cancer. This increased risk included most cancer sites examined, and was greatest following cancers of the breast, prostate, colon, and melanoma. Notably, the highest risk for thyroid cancers occurred within 1 year of the initial cancer diagnosis, although long-term ( $\geq 10$ years) NTC survivors remained at greater risk than the general population as well. These results were not categorized by specific thyroid tumor histologies.

Lal et al. (17) recently published a study confirming the greatly increased risk of subsequent thyroid cancer following 9 out of 10 primary non-thyroidal malignancies evaluated. The overall standardized incidence ratio (SIR) was most elevated in renal cancer (2.95), leukemia (2.24), lymphoma (2.15), lung cancer (1.99), and melanoma (1.69), and similar to previous studies, the risk was highest within the first year after diagnosis of the initial NTC $(12-14,17)$. This recurring observation is likely due to imaging obtained for staging purposes of the initial malignancy, as well as the increased clinical surveillance following diagnosis. In concordance with this theory, we found that patients with an antecedent or synchronous NTC were much more likely to be diagnosed with a thyroid mass incidentally on imaging compared to patients without an additional NTC.

Lal et al. (17) further divided the occurrences of secondary thyroid cancers into latency periods, finding that SIRs remained elevated 12-59 months after NTC diagnosis for nearly all cancers evaluated, with the exception of leukemia, uterine, and bladder cancers. Interestingly, the increased risk for developing thyroid cancer persisted 60-119 months after NTC diagnosis for renal (SIR 2.56) and breast cancer (SIR 1.16), with the elevated risk 
extending greater than 120 months (SIR 2.46) for renal cancer. Upon separation by histologic types, PTC had consistently higher overall SIRs than FTC or other thyroid cancer histologies, and one of the strongest associations observed in the study was between renal cancer and PTC (SIR 3.49), particularly within 2-11 months after NTC diagnosis (SIR 7.84). Correspondingly, renal cancer was the most common synchronous NTC in our study, although no cases of antecedent renal cancer were observed.

The occurrence of PTC following a primary NTC may be the consequence of treatment effect. Radiotherapy is a common treatment modality for numerous malignancies associated with an increased risk of secondary thyroid cancer. The thyroid gland may be included in the radiation field, particularly if the primary malignancy was head and neck cancer, lymphoma, leukemia, or potentially lung or breast cancer. However, many studies have demonstrated significant increases in the risk of thyroid cancer after primary NTC during latency periods that were unlikely to represent radiation-caused thyroid malignancies, with the understanding that thyroid cancer occurs at a average of 15 years after radiotherapy for a malignancy, and greater than 30 years after irradiation for benign disease (e.g. benign skin conditions) $(12-14,17,24)$. Furthermore, it has been shown that an increased risk of secondary PTC occurs in nonirradiated patients, and in many instances accounts for the majority of secondary PTC cases observed $(12,16,17)$.

In our study, only $35.0 \%$ of patients with an additional NTC had a history of radiation exposure. Given the recognized increased risk of thyroid cancer after numerous types of NTC in ours and other studies, it seems unlikely that the carcinogenic effects would be due to radiation alone. In addition, the 2-way relationship between thyroid cancer and other NTCs suggests a shared risk factor, perhaps a genetic susceptibility to multiple cancer types, or alterations in immune function after a primary cancer. The CHEK2 gene has been identified as a multisite cancer susceptibility gene, and when mutated is associated with cancers of the thyroid, breast, colon, prostate, and kidney (25). Future research may uncover additional genetic mutations responsible for multisite cancer syndromes associated with thyroid cancer.

A major limitation of this study is the retrospective method of data collection. We were limited in our identification of antecedent and synchronous NTCs by the medical history provided in the electronic medical record, and the true prevalence of additional NTCs may be higher than reported here. Alternatively, considering PTC is slow growing and may be present for many years prior to diagnosis, many patients classified as having synchronous PTC may in actuality have had PTC present for many years which went undetected until diagnosis of a primary NTC. In addition, the prevalence of PTC patients with an additional malignancy has not been typically reported in the literature, making it difficult to compare our results with others. Lastly, we were unable to perform a survival analysis due to our lack of consistent long-term post-operative follow-up in this cohort.

Despite the well-documented relationship between thyroid cancer, specifically PTC, and other NTCs, our study was the first to evaluate for potential differences in the clinicopathologic profile of these patients compared to those without additional NTCs. Based on our findings that these groups have similar primary tumor characteristics on presentation, we recommend that the management of PTC remain equivalent regardless of a whether or not a patient has a synchronous or antecedent NTC. In addition, we have shown that a significant number of patients with a primary NTC present with PTC incidentally on imaging for staging or surveillance, which is in concordance with the previous finding that the greatest risk of developing PTC after an NTC occurs within the first year of diagnosis. Surgeons should be aware of the frequency of synchronous PTC with these types of tumors and consider evaluation of the neck at the time of NTC diagnosis. 


\section{Acknowledgments}

Supported by a University of Wisconsin, Physician Scientist Training in Career Medicine grant, National Institutes of Health T32 CA009614-21.

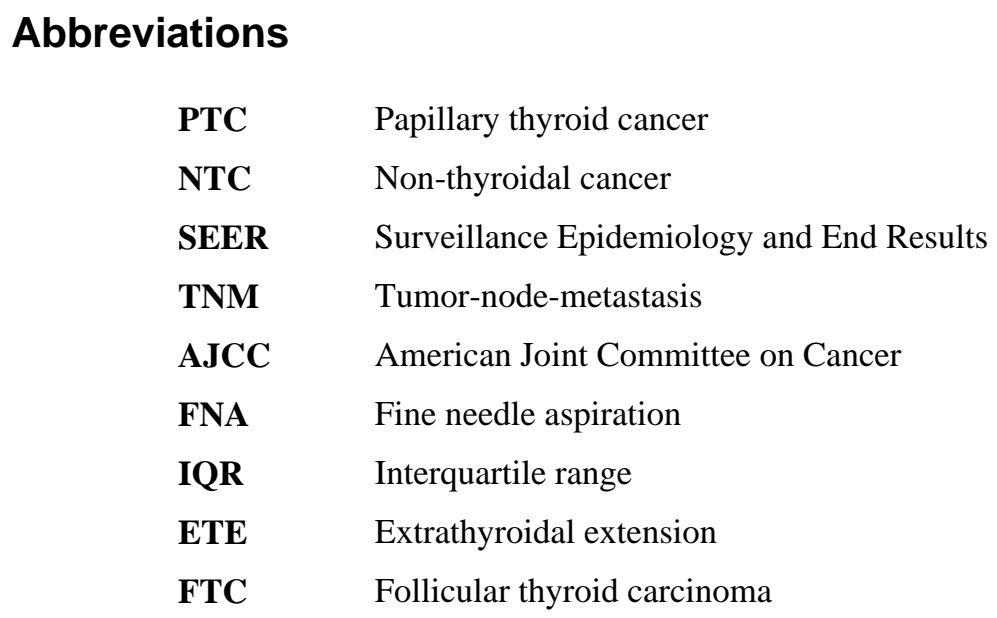

\section{References}

1. Howlader, N.; Noone, AM.; Krapcho, M., et al., editors. SEER cancer statistics review, 1975-2009. National Cancer Institute; Bethesda, MD: Available at: http://seer.cancer.gov/csr/ 1975_2009_pops09/ [Accessed October 10, 2012]

2. Davies L, Welch HG. Increasing incidence of thyroid cancer in the United States, 1973-2002. JAMA. 2006; 295:2164-2167. [PubMed: 16684987]

3. Ron E, Lubin JH, Shore RE, et al. Thyroid cancer after exposure to external radiation: a pooled analysis of seven studies of thyroid cancer. IV. Benign thyroid disease. Cancer Causes Control. 1999; 10:583-595. [PubMed: 10616827]

4. Franceschi S, Preston-Martin S, Dal Maso L, et al. A pooled analysis of case-control studies of thyroid cancer. IV. Benign thyroid diseases. Cancer Causes Control. 1999; 10:583-595. [PubMed: 10616827]

5. Repplinger D, Bargren A, Zhang Y, et al. Is hashimoto's thyroiditis a risk factor for papillary thyroid cancer? J Surg Res. 2008; 150:49-52. [PubMed: 17996901]

6. Eng C. Will the real Cowden syndrome please stand up: revised diagnostic criteria. J Med Genet. 2000; 37:828-830. [PubMed: 11073535]

7. Crial HW. Multiple primary malignancies arising in the rectum, brain, and thyroid; report of a case. US Nav Med Bull. 1949; 49:123-128.

8. La Vecchia C, Ron E, Franceschi S, et al. A pooled analysis of case-control studies of thyroid cancer. III. Oral contraceptives, menopausal replacement therapy and other female hormones. Cancer Causes Control. 1999; 10:157-166. [PubMed: 10231164]

9. Reynolds RM, Weir J, Stockton DL, et al. Changing trends in incidence and mortality of thyroid cancer in Scotland. Clin Endocrinol. 2005; 62:156-162.

10. Verkooijen HM, Fioretta G, Pache JC, et al. Diagnostic changes as a reason for the increase in papillary thyroid cancer incidence in Geneva, Switzerland. Cancer Causes Control. 2003; 14:1317. [PubMed: 12708720]

11. Leenhardt L, Bernier MO, Boin-Pineau MH, et al. Advances in diagnostic practices affect thyroid cancer incidence in France. Eur J Endocrinol. 2004; 150:133-139. [PubMed: 14763910]

12. Ronckers CM, McCarron P, Ron E. Thyroid cancer and multiple primary tumors in the SEER registries. Int J Cancer. 2005; 117:281-288. [PubMed: 15880372] 
13. Sandeep TC, Strachan MW, Reynolds RM, et al. Second primary cancers in thyroid cancer patients: a multinational record linkage study. J Clin Endocrinol Metab. 2006; 91:1819-1825. [PubMed: 16478820]

14. Sadetzki S, Calderon-Margalit R, Peretx C, Novikov I, Barchana M, Papa MZ. Second primary breast and thyroid cancers (Israel). Cancer Causes Control. 2003; 14:367-375. [PubMed: 12846369]

15. Bhatia S, Sklar C. Second cancers in survivors of childhood cancer. Nat Rev Cancer. 2002; 2:124132. [PubMed: 12635175]

16. Bhatti P, Veiga LH, Ronckers CM, et al. Risk of second primary thyroid cancer after radiotherapy for a childhood cancer in a large cohort study: an update from the childhood cancer survivor study. Radiat Res. 2010; 174:741-752. [PubMed: 21128798]

17. Lal G, Groff M, Howe JR, et al. Risk of subsequent primary thyroid cancer after another malignancy: latency trends in a population-based study. Ann Surg Oncol. 2012; 19(6):1887-1896. [PubMed: 22227921]

18. Canchola AJ, Horn-Ross PL, Purdie DM. Risk of second primary malignancies in women with papillary thyroid cancer. Am J Epidemiol Metab. 2006; 163:521-527.

19. Rubino C, de Vathaire F, Dottorini ME, et al. Second primary malignancies in thyroid cancer patients. Br J Cancer. 2003; 89:1638-1644. [PubMed: 14583762]

20. Brown P, Chen J, Hitchcock YJ, et al. The risk of second primary malignancies up to three decades after the treatment of differentiated thyroid cancer. J Clin Endocrinol Metab. 2008; 93:504-515. [PubMed: 18029468]

21. Edge, SB.; Byrd, DR.; Compton, CC., et al. AJCC Cancer Staging Manual. 7. New York, NY: Springer; 2010.

22. Vergburg FA, Mäder U, Tanase K, et al. Life expectancy is reduced in differentiated thyroid cancer patients $\geq 45$ years old with extensive local tumor invasion, lateral lymph node, or distant metastases at diagnosis and normal in all other DTC patients. JCEM. 2013; 98(1):172-80. [PubMed: 23150687]

23. Hartl DM, Leboulleux S, Al Ghuzlan A, et al. Optimization of staging of the neck with prophylactic central and lateral neck dissection for papillary thyroid carcinoma. Ann Surg. 2012; 255(4):777-83. [PubMed: 22418010]

24. Kikuchi S, Perrier ND, Ituarte P, et al. Latency period of thyroid neoplasia after radiation exposure. Ann Surg. 2004; 239:536-543. [PubMed: 15024315]

25. Cybulski C, Gorski B, Huzarski T, et al. CHEK2 is a multiorgan cancer susceptibility gene. Am J Hum Genet. 2004; 75:1131-1135. [PubMed: 15492928] 
Table 1

Comparison of Clinicopathologic Features of Patients with Papillary Thyroid Cancer With and Without a Synchronous or Antecedent Non-Thyroidal Cancer

\begin{tabular}{|c|c|c|c|}
\hline Variable & With NTC (n=60) & Without NTC (n=373) & p Value \\
\hline Age at PTC diagnosis, $y$, mean \pm SD & $56.4 \pm 15.5$ & $44.9 \pm 14.2$ & $<0.0001$ \\
\hline Female gender, n (\%) & $40(66.7)$ & $279(74.8)$ & 0.18 \\
\hline Radiation exposure prior to NTC diagnosis, $\mathrm{n}(\%)$ & $21(35.0)$ & $13(3.5)$ & $<0.001$ \\
\hline Presentation of PTC, n (\%) & & & $<0.001$ \\
\hline Neck mass & $28(46.7)$ & $308(82.6)$ & \\
\hline Incidental by imaging & $25(41.7)$ & $34(9.1)$ & \\
\hline Cervical lymph nodes & $1(1.6)$ & $14(3.7)$ & \\
\hline Distant metastases & $0(0.0)$ & $1(0.3)$ & \\
\hline Incidentally during surgery & $6(10.0)$ & $16(4.3)$ & \\
\hline Indication for surgery, $\mathrm{n}(\%)^{*}$ & & & 0.42 \\
\hline PTC on FNA & $39(65.0)$ & $227(60.9)$ & \\
\hline Suspicious for PTC on FNA & $1(1.7)$ & $29(7.8)$ & \\
\hline Neoplasm & $8(13.3)$ & $33(8.8)$ & \\
\hline Indeterminate FNA & $2(3.3)$ & $6(1.6)$ & \\
\hline Enlarging nodule/goiter & $6(10.0)$ & $42(11.2)$ & \\
\hline Symptomatic nodule/goiter & $4(6.7)$ & $32(8.6)$ & \\
\hline Graves' disease & $0(0.0)$ & $4(1.1)$ & \\
\hline PTC diameter, $\mathrm{cm}$, mean $\pm \mathrm{SD}$ & $1.7 \pm 1.4$ & $1.7 \pm 1.5$ & 0.96 \\
\hline Primary tumor, $\mathrm{n}(\%)^{\dagger}$ & & & 0.16 \\
\hline T1a & $26(43.4)$ & $155(41.6)$ & \\
\hline $\mathrm{T} 1 \mathrm{~b}$ & $12(20.0)$ & $93(24.9)$ & \\
\hline $\mathrm{T} 2$ & $5(8.3)$ & $62(16.6)$ & \\
\hline $\mathrm{T} 3$ & $15(25)$ & $50(13.4)$ & \\
\hline $\mathrm{T} 4 \mathrm{a}$ & $2(3.3)$ & $10(2.7)$ & \\
\hline Unknown & $0(0.0)$ & $3(0.8)$ & \\
\hline Lymph node metastases, n (\%) & & & 0.43 \\
\hline No & $43(71.7)$ & $293(78.6)$ & \\
\hline N1a & $7(11.6)$ & $38(10.2)$ & \\
\hline N1b & $10(16.7)$ & $42(11.2)$ & \\
\hline Distant metastases, $\mathrm{n}(\%)$ & $1(1.7)$ & $5(1.3)$ & 0.59 \\
\hline \multicolumn{4}{|l|}{ Histologic features of PTC, n (\%) } \\
\hline Extrathyroidal extension & $13(21.7)$ & $51(13.7)$ & 0.11 \\
\hline Lymphovascular invasion & $3(5.0)$ & $19(5.1)$ & 0.52 \\
\hline Multifocality & $24(40.0)$ & $146(39.4)$ & 0.92 \\
\hline Papillary thyroid microcarcinoma & $27(45.8)$ & $163(43.9)$ & 0.79 \\
\hline Follicular variant PTC & $11(18.3)$ & $64(17.2)$ & 0.82 \\
\hline
\end{tabular}




\begin{tabular}{|l|c|c|c|}
\hline Variable & With NTC $(\mathbf{n = 6 0})$ & Without NTC (n=373) & p Value \\
\hline Aggressive histologic type ${ }^{+}$ & $0(0.0)$ & $8(2.1)$ & 0.61 \\
\hline TNM Staging, $\mathrm{n}(\%)^{\dagger}$ & & & 0.001 \\
\hline Stage I & $38(63.4)$ & $309(82.8)$ & \\
\hline Stage II & $2(3.3)$ & $17(4.6)$ & \\
\hline Stage III & $12(20.0)$ & $25(6.7)$ & \\
\hline Stage IV & $8(13.3)$ & $22(5.9)$ & \\
\hline Total or completion thyroidectomy, n (\%) & $55(91.7)$ & $337(90.4)$ & 0.75 \\
\hline Radioiodine ablation, n $(\%)$ & $47(78.3)$ & $299(80.2)$ & 0.74 \\
\hline
\end{tabular}

Hurthle cell neoplasm or follicular neoplasm.

${ }^{\dagger}$ American Joint Committee on Cancer (AJCC) tumor-node-metatstasis (TMN) classification (21).

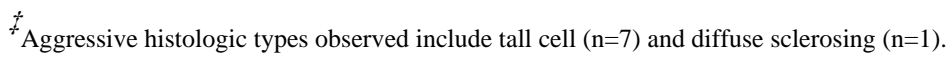

FNA, fine needle aspiration; NTC, non-thyroidal cancer; PTC, papillary thyroid cancer. 
Table 2

Observed Types and Frequencies of Synchronous or Antecedent Non-Thyroid Cancers in Patients with Papillary Thyroid Cancer

\begin{tabular}{|c|c|c|c|}
\hline Variable & $\begin{array}{c}\text { Synchronous or antecedent } \\
\text { NTC }(\mathbf{n}=60)\end{array}$ & Synchronous NTC $(n=17)$ & Antecedent NTC $(\mathrm{n}=47)$ \\
\hline Total observed cases of NTC * & 67 & 18 & 49 \\
\hline \multicolumn{4}{|l|}{ Types of NTC } \\
\hline Breast & 12 & 1 & 11 \\
\hline Prostate & 9 & 1 & 8 \\
\hline Melanoma & 8 & 3 & 5 \\
\hline Leukemia & 6 & 1 & 5 \\
\hline Lymphoma & 4 & 1 & 3 \\
\hline Renal cell carcinoma & 3 & 3 & 0 \\
\hline Cervical & 3 & 0 & 3 \\
\hline Oral cavity and pharynx & 3 & 2 & 1 \\
\hline Colon & 2 & 0 & 2 \\
\hline Testicular & 2 & 0 & 2 \\
\hline Pheochromocytoma & 2 & 1 & 1 \\
\hline Lung & 1 & 1 & 0 \\
\hline Esophageal & 1 & 1 & 0 \\
\hline Ovarian & 1 & 0 & 1 \\
\hline Dermatofibrosarcoma & 1 & 0 & 1 \\
\hline Lymphoepithelioma & 1 & 1 & 0 \\
\hline Bronchial carcinoid & 1 & 0 & 1 \\
\hline Islet cell tumor of pancreas & 1 & 0 & 1 \\
\hline Thymoma & 1 & 0 & 1 \\
\hline Medulloblastoma & 1 & 0 & 1 \\
\hline Osteogenic sarcoma & 1 & 0 & 1 \\
\hline Wilms tumor & 1 & 0 & 1 \\
\hline Gastrointestinal stromal tumor & 1 & 1 & 0 \\
\hline Neuroendocrine tumor of unknown primary & 1 & 1 & 0 \\
\hline
\end{tabular}

One patient had 2 synchronous NTCs. Two patients had 2 or more previous NTCs. Four patients had both a synchronous and antecedent NTC. NTC, non-thyroidal cancer. 